\title{
Estructura factorial exploratoria del capital humano intelectual
}

\author{
Eleazar Villegas-González ${ }^{1}$ \\ Arturo Sánchez-Sánchez² \\ Francisco Espinosa-Morales ${ }^{3}$ \\ Cruz García-Lirios ${ }^{4}$ \\ María L. Quintero-Soto ${ }^{5}$
}

Recibido 15 de marzo 2019 · Aceptado 30 de abril 2019.

\begin{abstract}
RESUMEN
A menudo, el capital humano intelectual emerge de climas laborales y liderazgos organizacionales centrados en la optimización de recursos y como una necesidad de innovar los procesos, con el fin de generar las oportunidades así como desarrollar y consolidar habilidades y conocimientos. En un escenario de flexibilidad laboral, el capital humano intelectual es considerado una respuesta de los talentos más que de los líderes y, por consiguiente, es de esperar una diversificación de capacidades. El objetivo del presente trabajo fue especificar un modelo para el estudio del proceso en comento. Se llevó a cabo una investigación no experimental, con una selección no probabilística de 300 empleados de microempresas del centro de México, área que se distingue por su grado de corrupción, inseguridad, contingencia y riesgos. A partir del ajuste de un modelo estructural, se aceptó la hipótesis nula relativa a la correspondencia entre los hallazgos teóricos y empíricos con respecto a la medición de relaciones entre indicadores y factores, pero solo se logró explicar un $67 \%$ de la varianza total, advirtiendo la inclusión de otros factores que la literatura identifica como "capital relacional e informacional".
\end{abstract}

Palabras claves: flexibilidad, clima, liderazgo, capital, rotación, salario.

\section{Exploratory Factorial Structure of Intellectual Human Capital}

\section{ABSTRACT}

Intellectual human capital often emerges from work environments and organizational leadership focused on the optimization of resources and the need to innovate processes,

I Doctor en Administración, Profesor de Tiempo Completo, UAEH, Pachuca. Contacto: eleazarvillegas@hotmail.com

2 Doctor en Administración, Profesor de Tiempo Completo, UAT, Tlaxcala. Contacto: arturossI@hotmail.com

3 Doctor en Administración, Profesor de Tiempo Completo, USON, Navojoa Contacto: fespinoz@navojoa.uson.mx

4 Estudios de Doctorado en Psicología, Investigador Asociado, ATN, Cuernavaca. Contacto: garcialirios@atn.mx

5 Doctora en Ciencia Política, Profesora de Tiempo Completo, UAEMEX, Neza. Contacto: quinluisa@yahoo.com.mx 
in order to create opportunities as well as develop and consolidate skills and knowledge. Within a flexible work scenario, intellectual human capital is considered to respond more to the talent than to the leaders and, therefore, a diversification of skills is to be expected. The objective of this article is to highlight a model for studying this process. A non-experimental research project was carried out with a non-probabilistic selection of 300 employees of small companies from central Mexico, an area known for its high level of corruption, insecurity, contingency and risk. Based on an adjustment to the structural model, the null hypothesis regarding the correspondence between theoretical and empirical findings and the measurement of relationships between indicators and factors was accepted, but only managed to explain $67 \%$ of total variance, suggesting the inclusion of other factors that literature identifies as "relational and informational capital."

Key words: flexibility, work environment, leadership, capital, turnover, salary.

\section{Introducción}

El objetivo del presente trabajo ha sido especificar un modelo para el estudio del capital humano en su dimensión informacional, con el fin de establecer las relaciones de dependencia reflejantes entre sus factores e indicadores.

El capital humano ha sido estudiado como inherente al liderazgo que se distingue por su comunicación y motivación vertical, aunque ha sido determinado por la flexibilidad en las condiciones, salarios y prestaciones laborales.

El capital humano, para los fines del presente trabajo, alude a capacidades, experiencias, habilidades y conocimientos. En ese sentido, es determinado por la flexibilidad laboral, la cual supone rotación e informalidad, y en la que la fuerza laboral ya no es dependiente de una oportunidad institucional u organizacional al diversificar sus opciones, compromisos, funciones y satisfacciones (García, Carreón \& García, 2017).

De este modo, la flexibilidad emerge de políticas económicas y estrategias tanto institucionales como organizacionales, y supone un contexto multifactorial. Las crisis económicas y los avances tecnológicos que afectaron a las organizaciones durante el siglo XX, en países centrales y economías periféricas, le dieron origen, minando las oportunidades del capital humano; pero el cambio climático supuso la reivindicación del capital humano, al establecer escenarios y 
sus efectos en la producción y consumo futuros (Piedrahita, Angulo \& López, 2013).

Entonces, la flexibilidad laboral está indicada por:

- Legislación ajustada al desempleo, con la finalidad de promover el crecimiento mediante subsidios y vacantes informales (García \& Bustos, 2012).

- Condiciones precarias en cuanto a la selección, adiestramiento y capacitación, reflejadas en la reducción de los salarios (García et al., 2016).

- La competencia desleal y el monopolio supusieron un aumento de las demandas y la disminución de la calidad de los procesos y productos (Carreón, 2016).

- Estrategias de exportación auspiciadas por el Estado y mano de obra especializada dispuesta a trabajar largas jornadas con bajo ingreso (García \& Bustos, 2012).

- Crisis energética y su efecto en la industria maquiladora, principalmente la automotriz, que incentivó la producción masiva sin control de calidad (García \& Bustos, 20I2).

- La contracción de la inversión pública y el desempleo masivo, que supuso la pauperización de los trabajos (García, Carreón \& Hernández, 2014).

- Proliferación de grupos de poder como barrera al emprendimiento y a la innovación, que enaltecieron la producción masiva sin calidad (García et al., 2015).

- Intervención estatal, que exacerbó las compensaciones por enfermedades y accidentes, así como el seguro de desocupación, que propiciaron la entrada de mano de obra no calificada (Carreón et al., 2016).

- Políticas públicas que generaron pobreza, contaminación y aglomeraciones de trabajadores en un solo sector industrialmaquilador (Carreón et al., 2016).

- Pactos corporativos, sindicales y estatales que produjeron corrupción, inequidad y movilización -sabotaje, huelgas, boicots, confrontaciones, mítines- (García, Bustos \& Carreón, 2017). 
No obstante, en el periodo que va de I945 a 1973 el crecimiento no solo fue centralista, sino además legitimó la rectoría del Estado e inhibió la participación de los sectores académicos y sociales en el desarrollo local y sustentable (Casas, 2003).

La ineficacia del modelo estatal supuso la emergencia de sectores informales que demandaron servicios públicos clientelares, a la vez que los sectores productivos fueron inhibidos por la burocracia proteccionista y sindicalista (Abreu, 2002).

Por consiguiente, al desmantelamiento de paraestatales se asociaron medidas desregulatorias de las relaciones obrero-patronales. Se trata de un cambio de gestión estatal a una selección personalizada de la fuerza laboral, la sustitución de maquinaria operada por colectivos y grupos a tecnologías automatizadas, que no solo permitieron extender la jornada laboral, sino además la trasformaron en continua y permanente, abriendo la posibilidad de empleo de personal no calificado y poco remunerado, subcontratado por un periodo corto (Alonso, 2008).

De este modo, la competencia entre las organizaciones que sustituyeron a las paraestatales y la emergencia de empresas en áreas de especialización e innovación subsecuentes a la revolución tecnológica, principalmente informacional, ha generado una demanda laboral de trabajos que, se estima, el $40 \%$ no existía hace cinco años (Awargala, 2009).

Si consideramos que los cambios ocupacionales obedecen a distintas dimensiones, entonces será posible advertir un escenario futuro en el que las ocupaciones se presentarán en función de las estructuras económicas, políticas, sociales, sindicales, educativas, científicas y tecnológicas. Sin embargo, en un sentido metodológico, el análisis de la flexibilidad laboral no se agota en sus causas sociohistóricas, económicas, políticas, laborales, sindicales, científicas o tecnológicas, sino además, en términos prospectivos, la dimensión académica es necesaria (López, 200I).

En este sentido, el establecimiento de las dimensiones informales respecto de las formales supone la incorporación de las dimensiones 
esgrimidas, pero con criterios e indicadores relativos a organizaciones, contratantes y buscadores de empleo (Guillén, 2007).

- Las empresas informales se distinguen de las empresas formales según (I) el tamaño de empleo y, o (2) el registro de la empresa y empleados. Estos límites se definen sobre una base nacional. En la India, las empresas con menos de 12 empleados se definen como informales. Los trabajos que, por definición, no existen: puestos de trabajo que existen, pero no son informales. El empleo en la economía informal, el empleo informal, el empleo informal fuera de la economía informal (Awargala, 2009).

- Las empresas informales excluyen los hogares que emplean a trabajadores domésticos remunerados (García et al., 2017).

- Los hogares que producen bienes para su propio uso final y hogares que emplean a trabajadores domésticos (Carreón et al., 2016).

- Trabajadores por cuenta propia poseen y operan una empresa en solitario o en colaboración con los miembros de la empresa, o uno adicional (García et al., 2017).

- Empleo de trabajadores familiares y empleados de forma ocasional, pero no sobre una base continua (Carreón, Hernández, Bustos \& García, 2017).

- Empleadores informales pueden emplear uno o más empleados en una base continua (Carreón et al., 2016).

De este modo, la flexibilidad, al estar circunscrita a la informalidad y a la formalidad, derivadas de las dimensiones sociohistóricas, económicas, políticas y organizacionales, resalta el enfoque prospectivo indicado por el grado de innovación científica y tecnológica que las organizaciones adoptarían, dependiendo de los contextos revisados (Morales, 2002).

Por consiguiente, la prospectiva de la flexibilidad laboral, entendida como un escenario probable de producción del conocimiento, con arreglo a fines de desarrollo tecnológico aplicado a la calidad de los procesos y los productos, supone algunas consecuencias esperadas a partir del periodo 2020-2060 (Adenike, 20I I). 
Se trata de un escenario en el que la migración llegaría un límite compartido con el nivel de productividad que se empezó a observar desde el año 2015, pero cuyos antecedentes se remontan a I950, cuando el Estado de Bienestar se consolidó y se inició la crisis de emprendimiento, innovación, productividad y competitividad (Peschner \& Fotakis, 2013).

Respecto de las consecuencias de orden educativo, principalmente en cuanto a la especialización ocupacional, la tendencia que se observó en 2010 no solo prevalece para la población económicamente activa, sino además se exacerbará en el año 2030 al estimar las asimetrías entre la dificultad de conseguir empleo (Kundu, 2007).

Es decir que a un aumento en las capacidades ocupaciones corresponde una reducción de los empleos elementales. En tal sentido, quienes no cuentan con una alta especialización y actualización de conocimientos están cercanos al desempleo, aunque quienes tienen posgrados no garantizan un empleo formal (Bautista, Delgado, García, Valdés, Hernández, Castro \& Trujillo, 2016).

Las oportunidades y las capacidades, desde la lógica de la flexibilidad ocupacional, son factores de equidad de género. A partir de 2030 no solo se esperará una paridad ocupacional entre hombres y mujeres, sino además se asumirá que el grado de educación-capacitación permitirá observar una distribución igualitaria en los puestos y responsabilidades de liderazgos (Peschner \& Fotakis, 2013).

Los indicadores relativos al nivel de selectividad migratoria, instrucción y capacitación avizorarán escenarios de equidad en el periodo que va de 2020 a 2040, pero asimétricos después de este lapso, dado el nivel de competitividad y dependencia tecnológica de las organizaciones (Maruko, 2012).

En este sentido, la literatura advierte que el impacto de la flexibilidad al ámbito de la salud ocupacional supone la incidencia de variables tales como la calidad de vida, el bienestar subjetivo, la cultura laboral, el clima organizacional y el capital humano -empatía, confianza, compromiso, emprendimiento, innovación, productividad, competitividad-(Carreón, 2016). 
Sin embargo, la literatura también constata la influencia del estrés -despersonalización, agotamiento, frustración- sobre el bienestar, la cultura-clima organizacional y el capital humano. De este modo, emerge la resiliencia como una respuesta personal, grupal y organizacional ante las amenazas y los riesgos que supone la implementación de la flexibilidad laboral en las organizaciones e instituciones (Luna, 2003).

En el caso de las instituciones de salud y educativas, el estrés asociado a la resiliencia genera absorción, dedicación y vigorización (Hermosa, 2006). Se trata de tres factores distintivos de los individuos, grupos y organizaciones que no solo desarrollan una resiliencia, sino además generan oportunidades y capacidades vinculadas a la satisfacción ocupacional (Meagher, 2003).

Sin embargo, la formación profesional del capital humano también está influida por factores emocionales, afectivos, sentimentales o heurísticos que determinan la toma de decisión y las estrategias de acción a seguir en caso de una contingencia. Es así como la desesperanza ha sido documentada como un estado emocional en el que los trabajadores se resignan no solo a sus condiciones laborales, sino además a su entorno laboral y familiar. Se trata de un proceso en el que la violencia es asumida como un factor ineludible, desde la búsqueda de empleo hasta la demanda de prestaciones en el trabajo (Omar, 2010).

En el caso de la hipermetropía, es considerada como una evasión emocional que inicia con la justificación de las condiciones laborales ante la flexibilidad laboral y culmina con la aceptación de despidos o sanciones distantes a los derechos humanos básicos y cercanos a la explotación (Peschner \& Fotakis, 2013).

Es posible apreciar que, en cualquiera de los casos, la flexibilización produce insatisfacción. Si se considera la trayectoria que se inicia con la flexibilización, prosigue con el compromiso, el emprendimiento, la innovación y culmina con la satisfacción, entonces se trata de un escenario optimista en el que las relaciones humanas y los avances tecnológicos se compaginan para reducir cargas laborales, incrementar salarios y, sobre todo, producir una identidad del trabajador para con la organización (Ramírez, 2005). 
Empero, la ruta que se inicia con la flexibilización, prosigue con la despersonalización, la desesperanza, la hipermetropía, y culmina con la insatisfacción, advierte un proceso individualizado en el que el trabajador se dedica a producir sin considerar la responsabilidad social de su desempeño (Carreón, García, Morales \& Rosas, 20I3). Por consiguiente, en ambas trayectorias se reflejan círculos viciosos en los que la insatisfacción prevalece, aunque reducida a su mínima expresión (Vargas, 20II).

La prospectiva de la flexibilidad ocupacional parece mostrar una correlación entre los avances científicos y tecnológicos respecto del aumento de las demandas de la calidad de los procesos y productos. A partir de esta relación, las oportunidades y las capacidades parecen orientarse desde criterios de especialización y actualización de conocimientos, indicadores del capital humano intelectual (Carreón et al., 2017).

Ello supone consecuencias sociales, organizacionales y personales cercanas al desempleo, exclusión y estrés de trabajadores migrantes con conocimientos y habilidades elementales, pero en el caso de aquellos con posgrados, no hay garantía de un empleo formal (Carreón, Morales, Rivera, García \& Hernández, 20I4).

En virtud de que la flexibilidad laboral es un lineamiento político institucional, el clima de relaciones que se edifica en las micro, pequeñas y medianas empresas del estudio tenderá a ser desfavorable al clima de tareas, apoyos e innovaciones, emergiendo el liderazgo como eje de decisión, comunicación y motivación de personal (Carreón et al., 2016).

Es cierto que el capital humano, al estar influido por la flexibilidad política, genera un entorno desfavorable para el equilibrio entre las demandas del entorno y las capacidades de las mipymes, así como anticíclico a la dinámica de las organizaciones, pero fortalece las ventajas competitivas de polivalencia o multifuncionalidad de la fuerza laboral, ya que tal sector se ajusta no solo a las políticas de empleo sino, además, a los requerimientos de la economía 4.0, la cual se distingue por su ambivalencia y escasez de oportunidades 
con las que las generaciones Millennials aprenden a desarrollar sus habilidades y conocimientos (García et al., 2017).

Los marcos empíricos destacan al liderazgo como factor de enlace entre el capital humano y la flexibilidad laboral, estableciendo que la comunicación bidireccional y la motivación intrínseca son indicativos de niveles y grados de demandas externas y recursos internos, ponderativas de equilibrios al momento de establecer un clima de relaciones, tareas, apoyos e innovaciones respecto de las condiciones, rotaciones, salarios, recompensas y prestaciones en situaciones cada vez más contingentes del mercado.

El liderazgo es un capital intangible en cuanto a habilidades, conocimientos y experiencias, los cuales no solo determinarán el clima sino, además, motivarán al personal hasta un punto tal que coexistirá un clima de relaciones con una rotación de funciones y disminución de salarios en situaciones de desempleo.

Carreón et al., (2017) advierten que las contingencias del mercado son determinantes indirectos del clima laboral y del desempeño de las organizaciones. Es decir, en la medida en que las políticas de fomento empresarial y microfinanciamiento incentiven la productividad, los líderes se ven comprometidos a llevar a cabo estrategias que, por su grado de improvisación, suponen una comunicación unidireccional y motivación en retribuciones que permita estar a la altura de las exigencias del mercado.

En tal escenario, el clima laboral es un factor mediador de las políticas económicas, productivas y de empleo, empero prevalece el liderazgo en el clima de relaciones laborales, siendo la flexibilidad un rasgo distintivo del entorno más que de la organización o de los grupos de trabajo al interior de ellas.

Carreón y García (2017) advierten que la influencia del liderazgo en la formación profesional del capital humano no solo implica un clima laboral que puede ser orientado a la flexibilidad laboral sino, además, supone la prevalencia del clima de relaciones sobre los demás tipos de climas de tareas, metas, innovaciones y apoyos, los cuales suponen 
un liderazgo tradicional que orienta a los empleados o subordinados, a la vez que motiva a los talentos y capitales intelectuales.

Si el clima laboral es resultado de las políticas locales y, a la vez, determina un tipo de desempeño informal y en rotación, entonces explicaría y anticiparía el éxito y la satisfacción de las organizaciones, al establecer un escenario de confianza y expectativas que podría influir en una formación profesional constante, capacitación especializada y automotivación al logro, las mismos que explican el tipo de liderazgo para las medianas empresas.

Si la influencia de la flexibilidad laboral sobre el capital humano intelectual descrita en la revisión de la literatura está de acuerdo con las observaciones que se realizarán en la investigación empírica, considerando el escenario de las microempresas del centro de México, que consiste en un alto grado de migración e inseguridad, entonces los marcos teóricos de la flexibilidad laboral suponen que el capital humano intelectual emerge del contexto de desregulación estatal, ya que el trabajador debe adquirir competencias laborales para poder reinsertarse al mercado laboral y mantenerse como optimizador de recursos e innovador de procesos. En tal sentido, la explicación de la literatura se ajustará a las observaciones realizadas en el contexto microempresarial del centro de México, donde la inseguridad y la corrupción prevalecen.

No obstante que la teoría de la flexibilidad advierte que un aumento de la desregulación estatal corresponde a un incremento de las habilidades y los conocimientos, experiencias e iniciativas de la fuerza laboral, las particularidades del contexto microempresarial sugieren una discrepancia que será explicada desde un análisis de la estructura de trayectorias de relaciones de dependencia entre las dimensiones del capital humano intelectual.

\section{Método}

Se realizó un estudio no experimental, transversal, exploratorio y correlacional. Se llevó a cabo una selección no probabilística de 300 empleados de microempresas del centro de México. El 67\% 
es de sexo femenino y el restante 33\% de sexo masculino. El 32\% culminó sus estudios de bachillerato, el 4I\% curso la licenciatura y el restante $27 \%$ cuenta con estudios de posgrado. El $45 \%$ declaró tener un ingreso inferior a 3.500 pesos mensuales $(M=34 \mathrm{I} 2 \mathrm{DE}$ $=23, \mathrm{I} 4)$, el 4I\% mencionó que sus ingresos oscilaron entre 3.500 y 7.000 pesos al mes $(M=58 \mathrm{I} 3 \mathrm{DE}=\mathrm{II} 3,24)$, y el restante $\mathrm{I} 4 \%$ reconoció que sus ingresos superaron los 7.000 pesos mensuales $(M=8 \mathrm{I} 24 \mathrm{DE}=234,56)$. El $42 \%$ está en matrimonio, el $24 \%$ en soltería y el restante $34 \%$ en unión libre.

Se utilizó la escala de Capital Humano Intelectual de Carreón (2017), la cual se edificó a partir de la técnica Delphi y Lickert, asumiendo que los reactivos de la literatura podrían ajustarse al contexto de estudio siempre que fuesen probados en muestras similares a las del estudio, así como la inclusión de opciones de respuesta que supusieron intervalos de significación en las respuestas de cada reactivo.

Se ponderaron las intenciones de los encuestados respecto de la informalidad y la rotación de personal. Es el caso del reactivo " $\mathrm{Si}$ hubiese desempleo, aprendería más habilidades para conservar mi oportunidad laboral". A cada ítem le corresponde alguna de las cinco opciones de respuesta: 0 = nada probable, $\mathrm{I}=$ muy poco probable, $2=$ poco probable, $3=$ algo probable y $4=$ muy probable.

Se midieron las necesidades y expectativas de los trabajadores respecto de sus líderes, estructura y condiciones laborales. Considérese el ítem: "Ante el desempleo, espero que mi jefe me asigne una función idónea". Cada reactivo incluye cinco opciones: $0=$ nada probable, $\mathrm{I}=$ muy poco probable, $3=$ algo probable y $4=$ muy probable.

Las encuestas se llevaron a cabo en las instalaciones de las mipymes, previa información de que los resultados del estudio no afectarían ni negativa ni positivamente su estatus laboral. Además, se garantizó por escrito el anonimato y la confidencialidad de los datos. La información fue procesada en el Paquete Estadístico para Ciencias Sociales (IBMSPSS-AMOS, por su acrónimo en inglés versión 20,0). Se estimó el parámetro alfa de Cronbach para la interpretación de la consistencia interna del instrumento, los estadísticos de adecuación y esfericidad de Bartlett y Kaiser Meyer Olkin para establecer la solución factorial, 
así como los pesos factoriales y el porcentaje de varianza explicada en un análisis factorial exploratorio de ejes principales con rotación promax, con el fin de poder establecer la validez del instrumento, la cual supone un constructo que emerge en contextos y muestras diferentes. Por último, se calculó el parámetro de correlación para establecer las probables trayectorias de relaciones entre los factores esgrimidos.

\section{Resultados}

La consistencia interna general del instrumento (alfa de 0,889) supera el mínimo requerido (alfa de 0,80). Ello quiere decir que la Escala de Capital Humano Intelectual puede aplicarse en contextos y muestras diferentes, arrojando resultados similares a los del presente estudio (véase tabla I). De hecho, si se asume como criterio de exclusión un mínimo requerido de 0,70 y un máximo de 0,90 , entonces ninguno de los ítems sería excluido por encontrarse en el rango ideal de consistencia esperada. 
Tabla I. Descriptivos del instrumento

\begin{tabular}{|l|c|c|c|c|c|c|c|c|c|}
\hline \multicolumn{1}{|c|}{$\mathrm{R}$} & $\mathrm{M}$ & $\mathrm{D}$ & $\mathrm{S}$ & $\mathrm{C}$ & $\mathrm{A}$ & & $\mathrm{F} 2$ & $\mathrm{~F} 3$ & $\mathrm{~F} 4$ \\
\hline$R l$ & 3,05 & $\mathrm{I}, 20$ & $\mathrm{I}, 06$ & $\mathrm{I}, 25$ & $0,7 \mathrm{II}$ & & & & \\
\hline$R 2$ & 3,08 & $\mathrm{I}, \mathrm{I} 4$ & $\mathrm{I}, 26$ & $\mathrm{I}, 59$ & 0,724 & & & & \\
\hline$R 3$ & 3,24 & $\mathrm{I}, \mathrm{I} 5$ & $\mathrm{I}, \mathrm{I} 3$ & $\mathrm{I}, 40$ & 0,752 & & & & \\
\hline$R 4$ & 3,26 & $\mathrm{I}, 28$ & $\mathrm{I}, 26$ & $\mathrm{I}, 44$ & 0,745 & & & & \\
\hline$R 5$ & 3,65 & $\mathrm{I}, \mathrm{I} 4$ & $\mathrm{I}, 26$ & $\mathrm{I}, 32$ & 0,762 & & & & \\
\hline$R 6$ & 3,16 & $\mathrm{I}, 92$ & $\mathrm{I}, 37$ & $\mathrm{I}, 07$ & $0,74 \mathrm{I}$ & & & & \\
\hline$R 7$ & 3,62 & $\mathrm{I}, 02$ & $\mathrm{I}, 73$ & $\mathrm{I}, 38$ & 0,706 & & & & \\
\hline$R 8$ & 3,42 & $\mathrm{I}, \mathrm{I} 4$ & $\mathrm{I}, 93$ & $\mathrm{I}, 26$ & 0,794 & & & & \\
\hline$R 9$ & 3,08 & $\mathrm{I}, \mathrm{I} 6$ & $\mathrm{I}, 02$ & $\mathrm{I}, 04$ & 0,702 & & & & \\
\hline$R l 0$ & $3, \mathrm{I} 5$ & $\mathrm{I}, 06$ & $\mathrm{I}, 26$ & $\mathrm{I}, 36$ & 0,762 & & 0,483 & & \\
\hline$R l 1$ & 3,67 & $\mathrm{I}, 84$ & $\mathrm{I}, 46$ & $\mathrm{I}, 36$ & $0,75 \mathrm{I}$ & & 0,484 & & \\
\hline$R l 2$ & 3,24 & $\mathrm{I}, 25$ & $\mathrm{I}, 35$ & $\mathrm{I}, 38$ & 0,705 & & $0,6 \mathrm{I} 8$ & & \\
\hline$R l 3$ & 3,59 & $\mathrm{I}, \mathrm{I} 3$ & $\mathrm{I}, 37$ & $\mathrm{I}, 30$ & 0,725 & & 0,703 & & \\
\hline$R l 4$ & 3,26 & $\mathrm{I}, 02$ & $\mathrm{I}, 32$ & $\mathrm{I}, 48$ & 0,732 & & 0,724 & & \\
\hline$R l 5$ & 3,20 & $\mathrm{I}, 43$ & $\mathrm{I}, 05$ & $\mathrm{I}, 28$ & $0,76 \mathrm{I}$ & & & 0,628 & \\
\hline$R l 6$ & 3,26 & $\mathrm{I}, 23$ & $\mathrm{I}, 0 \mathrm{I}$ & $\mathrm{I}, 03$ & 0,752 & & & 0,673 & \\
\hline$R l 7$ & 3,58 & $\mathrm{I}, 04$ & $\mathrm{I}, 07$ & $\mathrm{I}, 07$ & 0,762 & & & 0,694 & \\
\hline$R l 8$ & 3,52 & $\mathrm{I}, 04$ & $\mathrm{I}, 47$ & $\mathrm{I}, 44$ & $0,75 \mathrm{I}$ & & & 0,558 & \\
\hline $\mathrm{R} 19$ & 3,45 & $\mathrm{I}, \mathrm{I} 2$ & $\mathrm{I}, 59$ & $\mathrm{I}, 06$ & 0,786 & & & 0,643 & \\
\hline$R 20$ & $3,2 \mathrm{I}$ & $\mathrm{I}, \mathrm{I} 3$ & $\mathrm{I}, 30$ & $\mathrm{I}, 46$ & 0,724 & & & 0,733 & \\
\hline$R 21$ & 3,26 & $\mathrm{I}, 06$ & $\mathrm{I}, 03$ & $\mathrm{I}, 32$ & 0,752 & & & & 0,524 \\
\hline$R 22$ & 3,54 & $\mathrm{I}, \mathrm{I} 3$ & $\mathrm{I}, 02$ & $\mathrm{I}, 40$ & $0,75 \mathrm{I}$ & & & & $0,53 \mathrm{I}$ \\
\hline$R 23$ & $3,2 \mathrm{I}$ & $\mathrm{I}, 79$ & $\mathrm{I}, \mathrm{II}$ & $\mathrm{I}, 46$ & 0,704 & & & & 0,674 \\
\hline$R 24$ & 3,26 & $\mathrm{I}, \mathrm{I} 4$ & $\mathrm{I}, 72$ & $\mathrm{I}, 36$ & 0,736 & & & & $0,73 \mathrm{I}$ \\
\hline$R 25$ & 3,26 & $\mathrm{I}, 76$ & $\mathrm{I}, \mathrm{I6}$ & $\mathrm{I}, 36$ & 0,762 & & & & 0,732 \\
\hline$R 26$ & 3,54 & $\mathrm{I}, 02$ & $\mathrm{I}, 95$ & $\mathrm{I}, 26$ & 0,702 & & & & 0,743 \\
\hline$R 27$ & 3,27 & $\mathrm{I}, 53$ & $\mathrm{I}, 25$ & $\mathrm{I}, 46$ & $0,70 \mathrm{I}$ & & & & 0,853 \\
\hline$R 28$ & 3,06 & $\mathrm{I}, 20$ & $\mathrm{I}, \mathrm{I} 2$ & $\mathrm{I}, 39$ & 0,770 & & & & 0,743 \\
\hline
\end{tabular}

Fuente: elaboración propia, 2018.

Método de extracción: ejes principales, rotación: promax. Adecuación y esfericidad $\int \mathrm{X}^{2}=1864,322(300 \mathrm{gl}) \mathrm{p}=0,000 ; \mathrm{KMO}=0,857$ ( $\mathrm{M}=$ media, $\mathrm{DE}=$ Desviación Estándar, $\mathrm{S}=$ Sesgo, $\mathrm{C}=$ Curtosis, $\mathrm{A}=$ Alfa de Crombach quitando el valor del ítem. FI = Capital afectivo $(29,878 \%$ de la varianza total explicada $)$, F2 = Capital instrumental (17,973\% de la varianza total explicada ), F3 = Capital empático (II,47I\% de la varianza total explicada), F4 = Capital creativo (5,84\% 
de la varianza total explicada). Todos los reactivos incluyen cinco opciones de respuesta: $0=$ nada probable, $I=$ muy poco probable, $2=$ poco probable $3=$ algo probable y $4=$ muy probable.

La matriz de correlaciones muestra que existen relaciones positivas y significativas entre los cinco factores, evidenciado la posibilidad de una estructura reflejante del clima organizacional como factor de segundo orden (véase tabla 2).

Tabla 2. Correlaciones y covarianzas entre los factores

\begin{tabular}{|l|l|l|l|l|l|l|l|l|l|l|l|}
\hline & $M$ & $D$ & $N$ & $F l$ & $F 2$ & $F 3$ & $F 4$ & $F l$ & $F 2$ & $F 3$ & $F 4$ \\
\hline Fl & 29.7756 & 5.05445 & 205 & $\mathrm{I}, 000$ & & & & $\mathrm{I}, 893$ & & & \\
\hline F2 & 22.4078 & 4.25856 & 206 & $0,7899^{\ldots}$ & $\mathrm{I}, 000$ & & &, 342 & $\mathrm{I}, 892$ & & \\
\hline F3 & 17.8349 & 2.83280 & $2 \mathrm{I} 2$ & 0,832 & $0,744^{\ldots}$ & $\mathrm{I}, 000$ & &, 349 &, 325 & $\mathrm{I}, 932$ & \\
\hline F4 & 20.3173 & 3.29475 & 208 & 0,690 & 0,837 & 0,657 & $\mathrm{I}, 000$ &, 314 &, 435 &, 436 & $\mathrm{I}, 87 \mathrm{I}$ \\
\hline
\end{tabular}

Fuente: elaboración propia, 2018.

$\mathrm{M}=$ Media de cada factor, $\mathrm{D}=$ Desviación estándar de cada factor, $\mathrm{N}=$ Número de observaciones en cada factor, FI = Capital afectivo, F2 = Capital instrumental, F3 $=$ Capital empático, F4 = Capital creativo: $\mathrm{p}<0,0 \mathrm{I} ; \mathrm{p}<0,00 \mathrm{I} ;{ }_{\mathrm{p}}^{\mathrm{p}}<0,000 \mathrm{I}$.

Una vez establecidos los factores de primer y segundo orden, se procedió a establecer las relaciones de dependencia entre los factores del capital humano intelectual (véase tabla 3 ). 
Tabla 3. Relaciones de dependencia entre los factores

\begin{tabular}{|c|c|c|c|c|c|c|c|}
\hline Modelo & Hipótesis & Trayectoria & B & Sig & $\mathrm{R}$ & $\mathrm{R}^{2}$ & $\mathrm{R}_{\text {ajustada }}^{2}$ \\
\hline & $\mathrm{Ia}$ & $\begin{array}{l}\text { Capital afectivo } 6 \\
\text { Capital instrumental }\end{array}$ & 0,693 & 0,009 & 0,693 & 0,409 & 0,303 \\
\hline \multirow[t]{3}{*}{$I$} & $\mathrm{Ib}$ & $\begin{array}{l}\text { Capital afectivo } \leftarrow \\
\text { Capital empático }\end{array}$ & 0,644 & 0,000 & 0,634 & 0,488 & 0,384 \\
\hline & Ic & $\begin{array}{l}\text { Capital afectivo }< \\
\text { Capital creativo }\end{array}$ & 0,642 & 0,000 & 0,603 & 0,453 & 0,334 \\
\hline & $2 \mathrm{a}$ & $\begin{array}{l}\text { Capital instrumental } \leftarrow \\
\text { Capital afectivo }\end{array}$ & 0,570 & 0,260 & 0,550 & 0,323 & 0,207 \\
\hline \multirow[t]{3}{*}{ II } & $2 b$ & $\begin{array}{l}\text { Capital instrumental } \leftarrow \\
\text { Capital empático }\end{array}$ & 0,542 & 0,000 & 0,542 & 0,395 & $0,29 \mathrm{I}$ \\
\hline & $2 c$ & $\begin{array}{l}\text { Capital instrumental } \leftarrow \\
\text { Capital creativo }\end{array}$ & 0,592 & 0,000 & 0,592 & 0,342 & 0,238 \\
\hline & $3 a$ & $\begin{array}{l}\text { Capital empático } \leftarrow \\
\text { Capital afectivo }\end{array}$ & 0,470 & 0,000 & 0,49I & $0,24 \mathrm{I}$ & $0, \mathrm{I} 2 \mathrm{I}$ \\
\hline \multirow[t]{3}{*}{ III } & $3 b$ & $\begin{array}{l}\text { Capital empático } \leftarrow \\
\text { Capital instrumental }\end{array}$ & 0,452 & 0,320 & 0,419 & 0,270 & 0,150 \\
\hline & $3 c$ & $\begin{array}{l}\text { Capital empático } \leftarrow \\
\text { Capital creativo }\end{array}$ & $0,4 \mathrm{I} 2$ & 0,202 & $0,40 \mathrm{I}$ & 0,240 & $0, \mathrm{II} 5$ \\
\hline & $4 a$ & $\begin{array}{l}\text { Capital creativo } \leftarrow \\
\text { Capital afectivo }\end{array}$ & $0,33 \mathrm{I}$ & 0,002 & 0,383 & $0, \mathrm{I} 43$ & 0,084 \\
\hline \multirow[t]{2}{*}{ IV } & $4 \mathrm{~b}$ & $\begin{array}{l}\text { Capital creativo } 6 \\
\text { Capital instrumental }\end{array}$ & 0,332 & 0,067 & 0,357 & 0,184 & 0,072 \\
\hline & $4 c$ & $\begin{array}{l}\text { Capital creativo }< \\
\text { Capital empático }\end{array}$ & $0,32 \mathrm{I}$ & 0,002 & 0,356 & 0,194 & 0,084 \\
\hline
\end{tabular}

Fuente: elaboración propia, 2018.

$\beta=$ parámetro de relación de dependencia entre una variable dependiente y otra variable independiente, ambas en relación con otras variables determinantes. Significancia $=$ grado de error de atribución de la relación de dependencia, $\mathrm{R}=$ estadístico de relación de dependencia, $\mathrm{R}^{2}=$ estadístico de relación de dependencia elevado al cuadrado, $\mathrm{R}_{\text {aiustada }}^{2}=$ estadístico de relación de dependencia elevado al cuadrado y ajustado, reflejante de la varianza total explicada de cada modelo.

Es posible observar que el capital afectivo fue determinado por el capital instrumental $\left(\beta=0,693 ; p=0,000 ; R=0,693 ; R^{2}=0,419\right.$; $\left.\mathrm{R}_{\text {ajd }}^{2}=0,303\right)$. Respecto de las demás relaciones de dependencia, se observan valores bajos tendientes a relaciones espurias y además no significativas. 
Una vez establecidas las regresiones múltiples, se estableció un modelo estructural de trayectorias de relaciones de dependencia, en el que se pueden observar los indicadores y dimensiones del capital humano intelectual (véase figura I).

Figura I. Modelo estructural de trayectorias de relaciones de dependencia

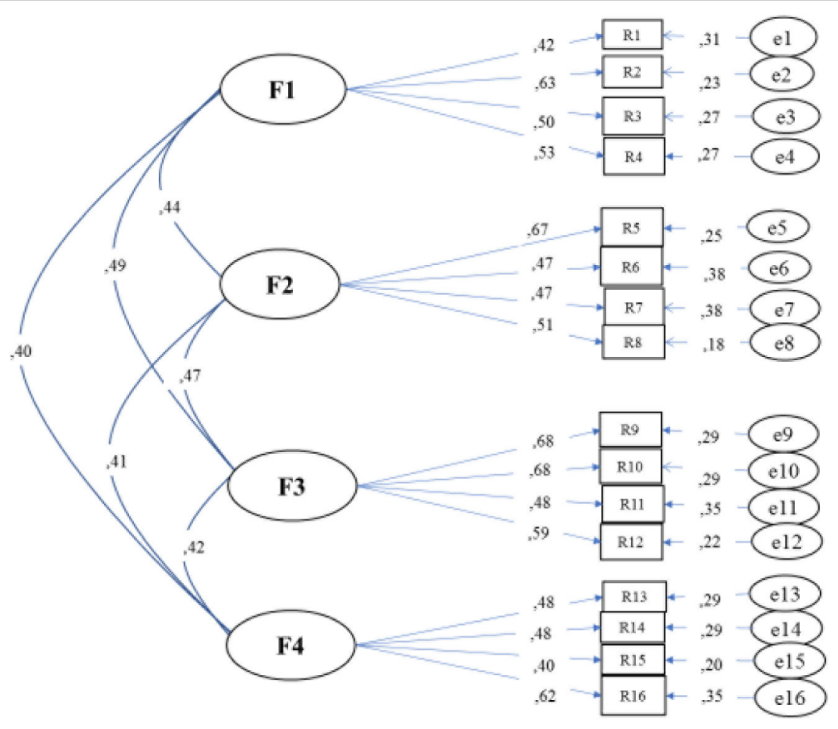

Fuente: elaboración propia, 2018.

FI = Capital afectivo, F2 = Capital instrumental, F3 = Capital empático, F4 = Capital creativo: $\mathrm{e}=$ Error de medición: $\cap$ relación entre factores, $\leftarrow$ relación entre error y reactivo, $\rightarrow$ relación entre factor y reactivo.

Es posible observar asociaciones moderadas entre los factores y de estos con sus indicadores, aunque solo el $62 \%$ de la varianza total fue explicada, con lo cual es menester la inclusión de otro factor relativo al capital humano intelectual relacional o informacional que podría incrementar la explicación. No obstante, los parámetros de ajustes $\int \mathrm{X}^{2}=5,552(2 \mathrm{gl}) \mathrm{p}=0,062 ; \mathrm{GFI}=0,974 ; \mathrm{NFI}=0,964 ; \mathrm{IFI}=$ 0,$977 ; \mathrm{CFI}=0,972 ; \mathrm{RMSEA}=0,009 \mathrm{~J}$ evidencian el ajuste de la estructura teórica respecto de las observaciones ponderadas. 


\section{Discusión}

El aporte del presente trabajo al estado de la cuestión radica en la especificación de un modelo para el estudio del capital humano intelectual en contextos de escasez de recursos, corrupción e inseguridad pública que, cuando menos, condicionan a las microempresas en alianzas estratégicas con las universidades públicas locales.

No obstante que el modelo exhibió un ajuste, el porcentaje de varianza total explicada advierte la inclusión de otros factores, que la literatura ha identificado como "capital relacional" y "capital informacional" para dar cuenta de las habilidades y los conocimientos vinculados a las relaciones públicas y al procesamiento de información. Ambos factores incrementarían el porcentaje de la varianza explicada si se considera que están orientados hacia la optimización de los recursos y la transformación de los procesos.

Pues bien, el presente estudio ha demostrado que el capital humano intelectual estaría configurado por cuatro factores, entre los que destaca el capital afectivo determinado por el capital instrumento, aunque el modelo advierte un ajuste en tanto ambas variables sean incluidas como factores asociados más que como factores determinantes.

Sin embargo, es necesario el contraste del modelo en un contexto en el que la flexibilización ha orillado a las microempresas a desarrollar estrategias de comunicación asertiva, con la finalidad de incrementar el capital humano y controlar los niveles de emotividad o afectividad ante la optimización de recursos o la innovación de procesos.

En este sentido, el capital instrumental que se distingue por su grado de manejo de recursos, herramientas, tecnologías, dispositivos o softwares se complementaría con el capital relacional, ya que el procesamiento de la información se realiza en tiempo real y con la participación de todos.

Por su parte, el capital empático podría asociarse con el capital social o el capital relacional, al potenciar el clima de relaciones 
como determinante de la motivación al logro de metas y objetivos, aunque reduciría al capital creativo, que se distingue por emerger en situaciones de crisis económica, política, social o educativa.

Precisamente, la relación entre el capital empático con el capital creativo supone un escenario de contingencia, riesgo e incertidumbre, en el que prevalecen las innovaciones de procesos y la diseminación de las propuestas conocidas como know bow, el cual orienta las iniciativas y evalúa su pertinencia en contextos diferentes a la microempresa.

\section{Conclusión}

El objetivo del presente trabajo ha sido especificar un modelo, mediante el establecimiento de la confiabilidad y la validez de un instrumento que mide el capital humano intelectual en cuatro dimensiones, a saber: afectiva, instrumental, empática y creativa, pero el porcentaje de la varianza total explicada advierte la inclusión de otros factores, como el capital relacional y el capital informacional, alusivos a la cooperación y el procesamiento de datos.

Pues bien, en un contexto de flexibilización laboral, la emergencia de cuatro factores del capital humano sugiere el desenvolvimiento de este en el ámbito microempresarial, el cual se diversifica en la medida en que las oportunidades escasean y la optimización de los recursos es insuficiente, animando a la creación de oportunidades mediante la innovación de los procesos.

\section{Referencias bibliográficas}

Abreu, M. (2002). Prospectiva de las organizaciones bursátiles en México. Denarious, 5, 3I-54.

Adenike, A. (201I). Organization climate as a predictor of employee job satisfaction. Business Intelligence Journal, 4, I5I-I66.

Alonso, P. (2008). Estudio comparativo de la satisfacción laboral en el personal de administración. Revista de Psicología del Trabajo y las Organizaciones, 24, 25-40.

Awargala, R. (2009). An economic sociology of informal work: the case of India. Research in the Sociology of Work, 18, 3I5-342. doi: 10.1108/S0277-2833 
Bautista, M., Delgado, M. A., García, C., Valdés, O., Hernández, G., Castro, F. J. y Trujillo, K. V. (2016). Contrastación de un modelo de cultura organizacional en centros de salud comunitaria ante la difusión informativa del VIH/SIDA desde el trabajo social. Enseñanza e Investigación en Psicología, 2 l(3), 248-255.

Carreón J. y García, C. (2017). Specification of a model for the study of vocational training and job. International Journal of Advances in Social Science and Humanities, 5(6), I3-I8.

Carreón, J. (2016). Desarrollo Humano: gobernanza y emprendimiento social. México: UNAM-ENTS.

Carreón, J., Bautista, M., García, C., Hernández, G., Sandoval, F. R., Pérez, G. y Valdés, O. (2016). Confiabilidad y validez de un instrumento que mide percepciones de salud ocupacional en migrantes de Huehuetoca, Estado de México. Obets, ll(2), 569-58I.

Carreón, J., García, C., Morales, M. y Rosas, F. (2013). Compromiso laboral en el Trabajo Social del sector salud. Invurnus, 8(2), I5-23.

Carreón, J., Hernández, J., Bustos, J. M. y García, C. (2017). Confiabilidad y validez de un instrumento que mide el compromiso de trabajadoras sociales en instituciones de asistencia sanitaria. Encrucijada, 26, 69-86.

Carreón, J., Hernández, J., Bustos, J. M. y García, C. (2017). Políticas de fomento empresarial y sus efectos sobre las percepciones de riesgo en caficultores de Xilitla, San Luis Potosí, centro de México. Poiesis, 32, 33-4I.

Carreón, J., Hernández, J., Quintero, M. L. y García, C. (2017). Confiabilidad y validez de un instrumento que mide la colaboración organizacional en una universidad pública de Huehuetoca, centro de México. Invurnus, 12(2), 9-17.

Carreón, J., Morales, M., Rivera, B., García, C. y Hernández, J. (2014). Emprendedurismo migrante y comerciante: Estado del conocimiento. Tlatemoani, 15, I-30.

Casas, R. (2003). Enfoque para el análisis de las redes y flujos de conocimiento, en M. Luna, Itinerarios del conocimiento: formas académicas y contenido. Un enfoque de redes (pp.19-50). Barcelona, España: Anthropos

García, C., Bustos, J. M. y Carreón, J. (2017). Dimensiones exploratorias del estigma social y laboral. Un estudio Delhi con estudiantes de pre-grado. Eureka, 14(I), 55-68.

García, C., Carreón, J. y Bustos, J. (2012). Socialización y compromiso laboral en trabajadoras sociales de instituciones públicas y privadas. Humanismo y Trabajo Social, 11, I77-192. 
García, C., Carreón, J. y Bustos, J. M. (2017). La salud ocupacional de unos adultos mayores en un contexto electoral de una localidad del centro de México. Doxa, 7(12), 36-49.

García, C., Carreón, J. y Hernández, J. (2017). Límites de los modelos de salud ocupacional. Estudio de adhesión al tratamiento del asma en trabajadores adultos mayores migrantes del Estado de México. Visión Gerencial, $26(\mathrm{I})$, I03-II8.

García, C., Carreón, J., Hernández, J. y Morales, M. (2014). Contraste de un modelo de compromiso laboral en centros de salud pública. Acta Universitaria, 24(I), 48-59.

García, C., Carreón, J., Hernández, J., Bautista, M. y Méndez, A. (2012). Modelamiento de variables sociopsicorganizacionales a partir de la revisión del estado del arte. Revista de la Facultad de Trabajo Social, 28(28), I3-60.

García, C., Carreón, J., Hernández, J., Carbajal, C., Quintero, M. L., Sandoval, F. R. y Valdés, O. (2016). Incidencia de las políticas de microfinanciamiento sobre la percepción de emprendimiento caficultor e implicaciones para el Trabajo Social. Ehquidad, 6, I I-36.

García, C., Valdés, O., Sánchez, R., Elizarraraz, G., Méndez, A. y Hernández, J. (2015). Diferencias entre emprendedores internautas con respecto a empatía, percepciones de riesgo y uso de aplicaciones tecnológicas. Prospectivas en Psicología, 2(I), 68-75.

Guillén, A. (2007). Globalización Neoliberal. México: UNAM.

Hermosa, A. (2006). Satisfacción laboral y síndrome de bournout en profesores de educación primaria y secundaria. Revista Colombiana de Psicología, 15, 8I-89.

Kundu, K. (2007). Development of the conceptual framework of the organizational climate. Vidyasagar University Journal of Commerce, 12, 100IIO.

López, E. (200I). ¿Liderazgo carismático en las organizaciones? Elementos para una reflexión sobre el cambio en las relaciones intra-organizacionales. Revista de Psicología Social, 16, 97-II5.

Luna, M. (2003). La red como mecanismo de coordinación y las redes de conocimiento. En M. Luna, Itinerarios del conocimiento: formas académicas y contenido. Un enfoque de redes (pp. 5I-78). Barcelona, España: Anthropos.

Maruco, M. (2012). Síndrome de Burnout en pediatras. Análisis de variables que podrían potenciar la aparición y agudización de los hospitales generales. Revista Académica de Ciencias Sociales, 16, I-9. 
Meagher, M. (2003). Organizaciones normativas: percepciones del sector académico y el empresarial acerca de sus relaciones con una red. En M. Luna. Itinerarios del conocimiento: formas académicas y contenido. Un enfoque de redes (pp. 259-305). Barcelona, España: Anthropos.

Morales, J. (2002). Decaimiento, mortandad y sobre vivencia organizacional: gestión estratégica de las organizaciones mexicanas en el neoliberalismo. Denarious, 5, 55-74.

Omar, A. (2010). Liderazgo transformador y satisfacción laboral: el rol de la confianza en el supervisor. Liberabit, 17, I29-137.

Peschner, J. y Fotakis, C. (2013). Growth potential of EU human resources and policy implications for future economic growth. Working Paper European Comission. DOI: I0.2767/79370

Piedrahita, D., Angulo, H. y López, H. (2013). Flexibilidad laboral, análisis conceptual y su incidencia en los países latinoamericanos, recientes debates. Prospectiva, 28, 221-242.

Ramírez, H. (2005). El conocimiento como arma competitiva entre empresas, en P. Dorantes, E. Espinosa, I. Guillen, F. Martínez, L. Ureña, y C. Valladares, Análisis, evaluación y perspectiva de la administración (pp. II7-I35). México: UAM.

Vargas, J. (20II). Organización del trabajo y satisfacción laboral: un estudio de caso en la industria del calzado. Revista Electrónica Nova Scientia, 4, 172-204. 